\title{
Structures of Binary Star Coronae
}

\author{
Nancy S. Brickhouse \\ Harvard-Smithsonian CfA, \\ 60 Garden St., Cambridge, MA 02138 USA
}

\begin{abstract}
.
Stellar coronae in binary star systems offer both a puzzle and an opportunity. We might expect that large magnetic loop structures on close binaries, such as RS CVn systems and contact binaries, would show evidence for interactions between the stars. While some radio studies support this scenario, there is surprisingly little evidence from EUV and $\mathrm{X}$-ray observations for differences between binary and single star systems. Meanwhile, the binary systems offer observational opportunities through rotational modulation and eclipses of flaring and non-flaring regions. Localizing the sources of coronal emission is key to making the magnetic connection to the underlying photosphere. We discuss the structure of stellar coronae from the perspective of studies of binary systems.
\end{abstract}

\section{Introduction}

Are coronae in binary systems fundamentally different from the coronae in comparable single stars? Or do binary systems simply offer us an interesting array of analysis tricks from which we can study the generic properties of stellar coronae?

From the perspective of X-ray studies, the role of binarity in the formation of X-ray emitting coronal structures appears minimal. While high signal-tonoise spectra exist only for a small sample of active systems, no glaring differences arise between active binaries and their single counterparts, once rotation is accounted for. Comparing luminosity functions for larger samples of binary systems and single stars suggests subtle differences not easily explained except in an evolutionary context. Meanwhile, radio data show clear evidence for interbinary emission, and possibly interaction. Should we expect extended, interacting magnetic fields to affect $\mathrm{X}$-ray emission as well as radio emission?

In this paper I will first briefly review these disparate pieces of evidence, and then proceed to describe some recent observations of X-ray emission from binaries. I will focus on various techniques that are being applied to determine fundamental characteristics of the coronal structures: how big? how hot? how dense? and located where?

Additional observational clues may also be useful to establish patterns of coronal structure. For example, time-variability studies indicate that some structures are highly prone to flaring or show evidence for cycles, while others do not. Coronal abundance patterns for active binaries are quite different from the Sun (e.g. Drake et al. 2001). Tying these more indirect clues to theoretical models 
of magnetic confinement, heating, and stability of coronal plasmas will require major theoretical effort, as we do not yet know the physical processes that cause flaring or abundance fractionation. In this paper I will not discuss these indirect clues, but will focus instead on quantifying the physical conditions. Additional details are available in the recent review by Favata \& Micela (2003).

There is a growing consensus that active stars have coronal structures that are significantly different from the structures on less active stars. These active coronae are not simply Suns extrapolated to higher temperatures or larger filling factors, but seem to be exhibit new phenomena. Recent observational evidence perhaps helps explain why we do not see a stronger effect from binarity.

\section{Evidence that Binary Coronae are Special}

\subsection{Luminosity Functions and Binarity}

Although rotation is the main parameter controlling the X-ray luminosity, evolution also plays a role. In the Hyades cluster, $\mathrm{dK}$ and $\mathrm{dM}$ binary components are more X-ray luminous than their single star counterparts (Pye et al. 1994; Stern, Schmitt, \& Kahabka 1995). While the lower mass stars spin down more slowly, and thus a larger population of fast rotators might be expected, this effect is apparently due instead to long period ( $>1 \mathrm{yr}$ ) systems. These authors suggest that the binary companion modifies or disrupts the pre-main sequence disk, affecting the distribution of angular momentum among stars approaching the main sequence. More recently, Franciosini, Randich, \& Pallavicini (2003) have shown that the Praesepe binary and single star luminosity functions, unlike those in the Hyades at a similar age, are indistinguishable from each other. These authors conclude that age alone does not control rotational evolution, and suggest that the history of the cluster itself may also affect the development of coronae.

\subsection{Finding Interbinary Emission}

Strong coronal activity in binary systems such as RS CVn leads to the natural suggestion that the magnetic field lines from the two stars connect and interact. The model of Uchida \& Sakurai (1985) provides a detailed example of how the large scale magnetic structures might connect to localized active regions. Very long baseline interferometry (VLBI) measurements of many different systems indicate extended radio emission, especially associated with flare decays (e.g. Lestrade et al. 1988; Mutel et al. 1985). Siarkowski et al. (1996) concluded from comprehensive ASCA X-ray light curve analysis of AR Lac that compact and well-localized emission regions existed on both stars, with additional extended emission sources likely, though not required.

While indirect evidence from X-ray light curves and emission measures and from radio flux density, polarization and spectral index measurements have supported extended emission, only recently have spatial resolution and sensitivity allowed direct determination of extended emission. Beasley \& Güdel (2000) have resolved a large quiescent radio-emitting region between the two stars of UX Ari. The detection of such a large stable structure between the stars raises the question of whether magnetic loops from two stars are actually interacting, 
or whether the loop is instead a large structure based in one component. Meanwhile, extended coronal X-ray emission, as suggested for AR Lac, has yet to be determined from Chandra or XMM-Newton, although evidence for small loops is accumulating.

\section{Modeling Coronal Structure from X-ray Data}

\subsection{The X-Ray/Radio Connection: Thermal versus Non-thermal Emission}

The strong correlation between X-ray emission and radio emission for many classes of active stellar systems suggests a relationship between coronal heating and the fast particle acceleration mechanism (see Güdel 1994, and references therein). On the Sun, spikes of non-thermal hard X-ray (10 to $100 \mathrm{keV}$ ) emission and gyrosynchrotron radio emission from fast particles, which are accelerated in reconnecting regions, indicate the trigger of a solar flare. The flare is consequently observed as a rise in thermal soft X-ray emission, as the bulk of the plasma is evaporated from the chromosphere and heated. The trigger plasma and subsequent flaring loop are spatially separated, but presumably connected by the evolving magnetic fields. Known as the Neupert effect in the solar flare context, the correlation between radio and X-ray emission is less well understood in the stellar context, particularly for quiescent emission. Perhaps all heating and acceleration can be described by "flaring" over a wide range of scales. It is also possible that some quiescent emission may arise from highly stable magnetic structures with steady continuous heating.

In the stellar case the radio and X-ray emissions from a given system often arise from different plasma emission mechanisms and from physically separated magnetic structures. Thermal X-ray emission is dominated by the highest density plasma, and thus is most likely to arise in structures confined by strong magnetic fields. At very high temperatures line radiation decreases as the ions become fully stripped and bremsstrahlung becomes the only thermal X-ray emission process. Only at these high temperatures and/or low densities can radio emission compete. Emission measure distributions from X-ray spectra rarely indicate plasma temperatures greater than $\sim 50 \times 10^{6} \mathrm{~K}$ in quiescence, though some of the largest, hottest flares approach temperatures consistent with nonthermal radio emission and very high energy radiation. The flare observed on II Peg with Chandra HETG (Huenemoerder, Canizares, \& Schulz 2001) is an example of such an extreme case.

\subsection{Emission Measure Diagnostics}

Figure 1 shows emission measure distributions for two active binaries in comparison with Procyon and the Sun (Brickhouse \& Drake 2000). While Procyon is more luminous than the Sun, its emission measure distribution has a similar shape. On the other hand, HR 1099 and Capella are remarkably different from the Sun. Both systems show a peak at $6-8 \times 10^{6} \mathrm{~K}$, enhanced above the minimum near $10^{6} \mathrm{~K}$ by at least two orders of magnitude. At higher temperatures, the emission measure for HR 1099 rises again, while it falls to a plateau for Capella. Magnetic loop models originally developed for the solar case can be 
applied to these active binaries, if the loop cross section is allowed to expand significantly with height (Schrijver, Lemen \& Mewe 1989); such loops are large, with scale heights the order of a stellar radius, much larger than high temperature solar loops imaged with Yohkoh (see http://www.lmsal.com/SXT).

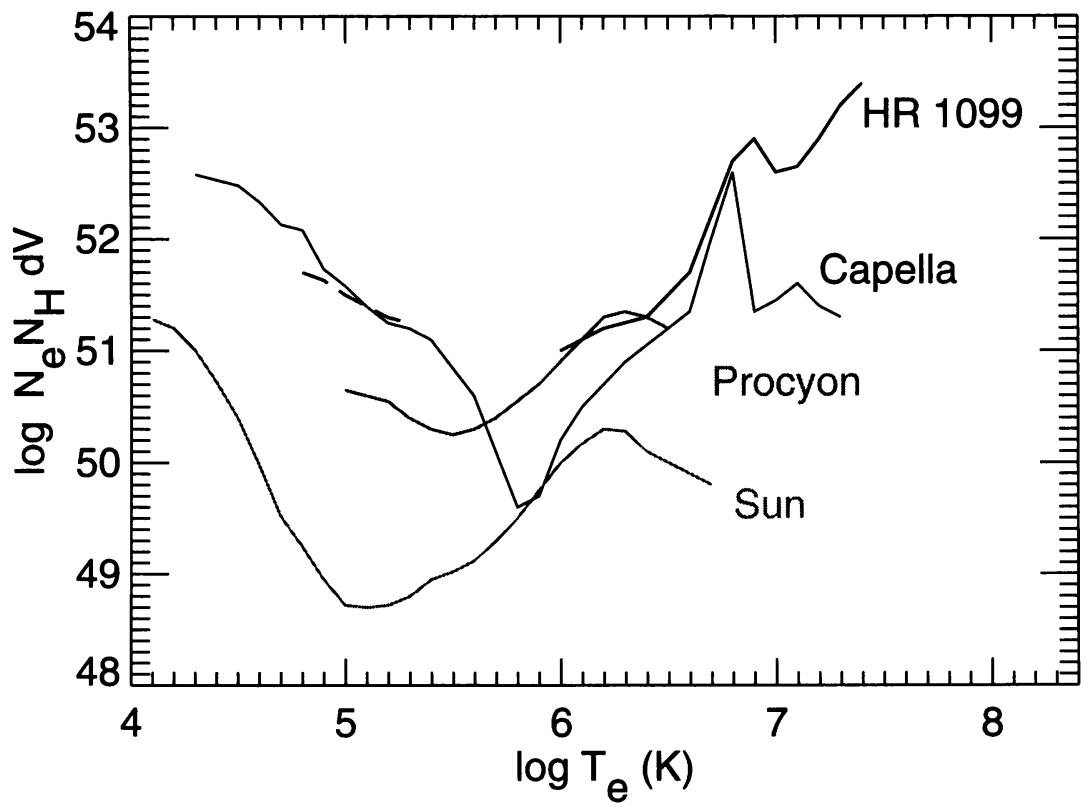

Figure 1. Emission measure distributions for the active binaries Capella and HR 1099 compared with Procyon and the Sun. Data for models are from Brickhouse (1996) for Capella; Griffiths \& Jordan (1998) for HR 1099; Drake, Laming, \& Widing (1998) for Procyon; and Raymond \& Doyle (1981) for the Sun.

Active binaries dominate the sample of EUV and X-ray spectra, but some rapidly rotating single stars have also been observed. Sanz-Forcada, Brickhouse, $\&$ Dupree (2003) have compared emission measure distributions of the cool star binaries in the EUVE archive with the small sample of single stars, and found no differences in the structure properties of bright active coronae between binary and single star systems, once rotation is taken into account.

\subsection{Line Flux Density Diagnostics}

Independent measurements of the electron density, in conjunction with the emission measure, set tight constraints on the scale sizes of the magnetic loops. Dupree et al. (1993) found evidence for densities exceeding $10^{12} \mathrm{~cm}^{-3}$ in Capella; however, densities from EUVE have not been viewed as definitive, given the difficulties with continuum subtraction, potential line blending, and atomic data uncertainties (see Brickhouse 1996). Chandra and XMM-Newton grating observations require careful attention as well. In Capella the density from O VII line 
ratios is now consistently derived from several observations with good signal-tonoise, and is $\sim 2 \times 10^{10} \mathrm{~cm}^{-3}$, suggesting a loop height of only a few percent of the stellar radius (Canizares et al. 2000; Brickhouse 2002).

Additional diagnostics from the Chandra gratings provide mixed support for still higher densities at $\sim 10^{7} \mathrm{~K}$. Figure 2 shows a portion of the Chandra LETG spectrum of Capella. The ratio of the Fe XIX lines $(\lambda 91.02 ; \lambda 108.37)$ provides compelling evidence for density $>10^{12} \mathrm{~cm}^{-3}$ (Brickhouse 2002), while Ne IX triplet lines indicate densities below $\sim 10^{11} \mathrm{~cm}^{-3}$ (Ness et al. 2003). Given FUSE and HST measurements showing that Capella's two stars both contribute to the high temperature emission (Johnson et al. 2002, and references therein), it is possible that apparently inconsistent densities from line ratios result from combining flux from different types of structures. The high density indicated from Fe XIX correlates with the peak of the emission measure distribution, suggesting pressures three orders of magnitude greater than for solar active regions. If this density is correct, then some physical processes must be missing from our current understanding of magnetic heating and confinement.

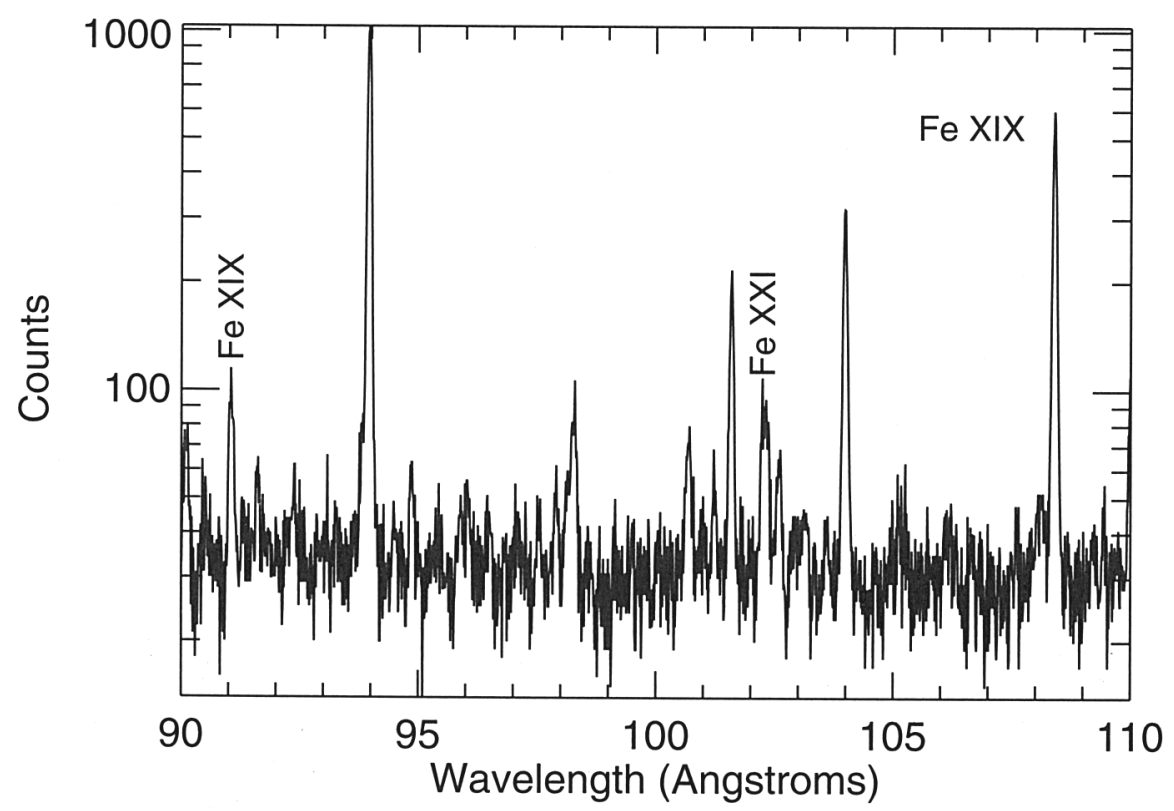

Figure 2. Chandra LETG/HRC-S spectrum of Capella, showing useful density-sensitive lines. The ratio of the Fe XIX lines indicates a density above $\sim 10^{12} \mathrm{~cm}^{-3}$, using the APEC models of Smith et al. (2001). The Fe XXI line shown forms a density-sensitive pair with $\lambda 128.73$ (not shown), but needs to be carefully deblended from O VIII $\mathrm{H}_{\alpha}$ lines. The data shown are summed over 6 observations for a total exposure time of $234.2 \mathrm{ksec}$. 


\section{Exploiting the X-ray Binary}

If binarity does not grossly affect the X-ray emitting corona of active stars, then the additional diagnostics available from binary systems can provide general information on cool star coronal structures.
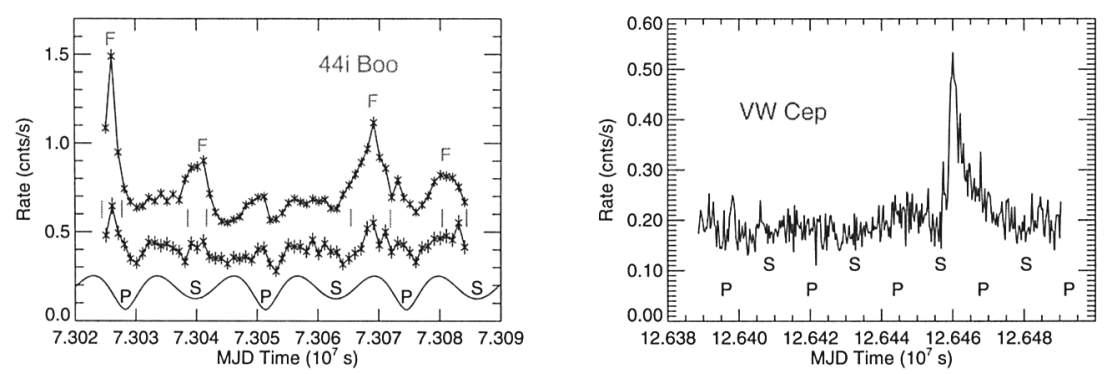

Figure 3. Light curves of 44i Boo (left) from Chandra HETG/ACIS-S and VW Cep (right) from Chandra LETG/HRC-S. Upper curve for 44i Boo is the first order light curve, middle curve is filtered for emission between 14 and $20 \AA$ and multiplied by a factor of 3 (adapted from Brickhouse et al. 2001). Lower curve is the optical light curve with primary and secondary eclipses marked. Flare times are marked. VW Cep curve is for zero-order only (Hoogerwerf, Brickhouse, \& Dupree 2003).

\subsection{Flare decay modulation}

Flare decay modulation can provide a geometrical constraint on the size of the decaying magnetic loop. Choi \& Dotani (1998) used the ASCA light curve to measure a flare decay on the primary star of the eclipsing contact binary VW Cep. They estimated a loop height slightly smaller than the size of the secondary and inferred a density of about $5 \times 10^{10} \mathrm{~cm}^{-3}$.

A long-duration, extremely hot flare on the eclipsing binary Algol observed by Beppo-Sax was totally eclipsed by the primary star, allowing a geometrical determination of the size of the emitting region (Schmitt \& Favata 1999). Favata \& Schmitt (1999) compare magnetic loop lengths derived from models with these geometrical constraints and find that the models overestimate the loop height. Assuming that flares decay through a series of quasi-static loops undergoing radiative cooling (van den Oord \& Mewe 1989) Favata \& Schmitt derive a loop length of $\sim 2 \times 10^{12} \mathrm{~cm}$ with a plasma density of $10^{10} \mathrm{~cm}^{-3}$. A newer method tested on Yohkoh observations of solar flares uses the slope of the temperature vs density relationship during the flare decay (Reale 1997). Assuming that the volume of the flare remains constant, but allowing for input heating during the flare, the square root of the emission measure can serve as a proxy for density. This method yields a loop length for the Algol giant flare of $8 \times 10^{11} \mathrm{~cm}$. Over the range of values allowed by these methods, the loop length is between 1.2 and 7 times the stellar radius, compared with $\sim 0.6$ times the stellar radius 
determined geometrically. Thus loops may be generally smaller than typically derived from X-ray flare decay techniques.

\subsection{Resolving the light curves of the two components}

Given two extremely similar stars in a binary system, how similar or different do we expect their coronae to be? New Chandra observations resolve the two dM5.5e stars of UV Ceti and provide individual light curves (Audard, Güdel, \& Skinner 2003). While the two stars have similar low level luminosities and temperatures, the $\mathrm{B}$ component shows a higher $\mathrm{X}$-ray flare rate and thus nearly twice the average $\mathrm{X}$-ray luminosity as the $\mathrm{A}$ component. In quiescence the radio emission of the B component is much stronger. During flares the B component emission is only moderately polarized, while on the A component the flares are circularly polarized, possibly produced by a coherent cyclotron maser (see Bingham, Cairns, \& Kellett 2001) Studies of such "twins" in binary systems demonstrate intrinsic diversity among coronal structures.

\subsection{X-ray spectral line widths}

New Chandra grating observations of the active eclipsing binary AR Lac show broadened line profiles at quadrature, consistent with both stars being active and emission concentrated near their photospheres (Huenemoerder et al. 2003). Although limited phase coverage prevents eclipse detections, the profiles during conjunction do not appear broader than instrumental. The high variability of this system makes it difficult to infer coronal structure from light curve analysis alone, which is unfortunate given that AR Lac is one of the X-ray brightest, totally eclipsing RS CVn systems. Even without detailed coronal structure maps, the new line profiles are difficult to reconcile with the highly extended emission suggested by Siarkowski et al. (1996).

\subsection{Tomography using the eclipse by a "dark star"}

Güdel et al. (2003) have reported new XMM-Newton light curve measurements of the $\alpha \mathrm{CrB}$ system during eclipse. This system consists of a G5 V main sequence star in an eccentric orbit $(17.4 \mathrm{~d})$ with an X-ray dark A0 primary. The primary eclipse is total, and thus with the high sensitivity of the XMM CCDs, the ingress and egress portions of the light curve can be used to map in some detail the coronal structure. Although such reconstructions are non-unique, the authors use a number of different approaches with consistent results. The G5 star appears to have a similar distribution of X-ray brightness to that of the Sun, despite having 30 times the X-ray luminosity. The corona is hotter $\left(5 \times 10^{6} \mathrm{~K}\right)$ and appears more dense than the Sun in the inferred active regions.

We also note Fred Walter's report in these proceedings on measurements of the eclipse of the K dwarf corona in V471 Tau by its white dwarf companion. In this case the white dwarf can probe the outer atmosphere of the $\mathrm{K}$ dwarf through absorption.

\subsection{X-ray Doppler imaging with light curves and velocity profiles}

X-ray Doppler imaging (albeit very crude compared to the optical!) is now possible in the X-rays. Chandra HETG observations of the partially eclipsing 
contact binary 44i Boo show evidence for two localized active regions on the primary star (Brickhouse, Dupree, \& Young 2001). Emission region location and size constraints are determined from the light curve (Fig. 3). A light curve constructed from spectral regions formed near the emission measure distribution peak (i.e. a light curve constructed predominantly from strong lines), and excluding flare times, shows phase-dependent modulation. Of particular interest are three reproducible narrow dips that are associated with emission near the pole of the primary as it rotates out of view. The active region must have an extremely compact latitudinal extent. Line profile centroid shifts indicate velocity changes of $180 \mathrm{~km} \mathrm{~s}^{-1}$ and follow the orbital velocity of the primary (Fig. 4).

By summing line profiles of different emission lines, the signal-to-noise ratio can be greatly increased. Assuming that the emission lines are roughly co-spatial, one can then determine more parameters (in addition to the line centroid) associated with the line profile shape. Figure 5 illustrates the potential of such a technique.
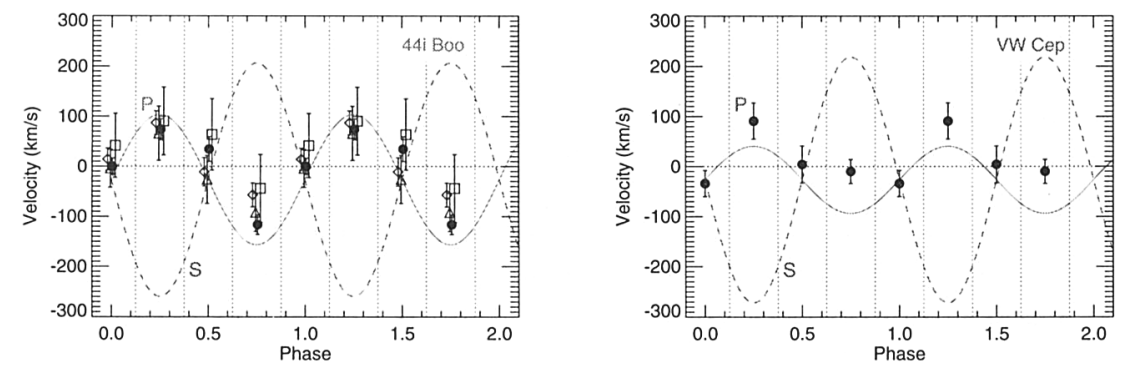

Figure 4. Velocity measurements from emission line centroid shifts as functions of orbital phase for 44i Boo (left) and VW Cep (right). Radial velocity curves are overplotted with primary star (solid) and secondary star (dashed). For 44i Boo the symbols with error bars are from individual line measurements. For VW Cep four emission lines have been co-added. The VW Cep analysis is preliminary, pending refinement of the LETG/HRC-S wavelength scale.

New Chandra LETG observations of the contact binary VW Cep show some evidence for line centroid shifts, but no reproducible light curve modulation is apparent over the five epochs of the observation (Figs. 3 and 4). Further analysis of 44i Boo, VW Cep and other systems is underway (Hoogerwerf, Brickhouse, \& Dupree 2003, in preparation).

\section{Conclusions}

New X-ray studies of active binaries are providing a wealth of information about the diversity of coronal structure. We have argued that binarity itself is unlikely to be the major factor determining what kinds of coronal structures can exist. While interacting magnetic fields between the stars can lead in principle to structures unique to binaries, such structures are more likely to produce non- 

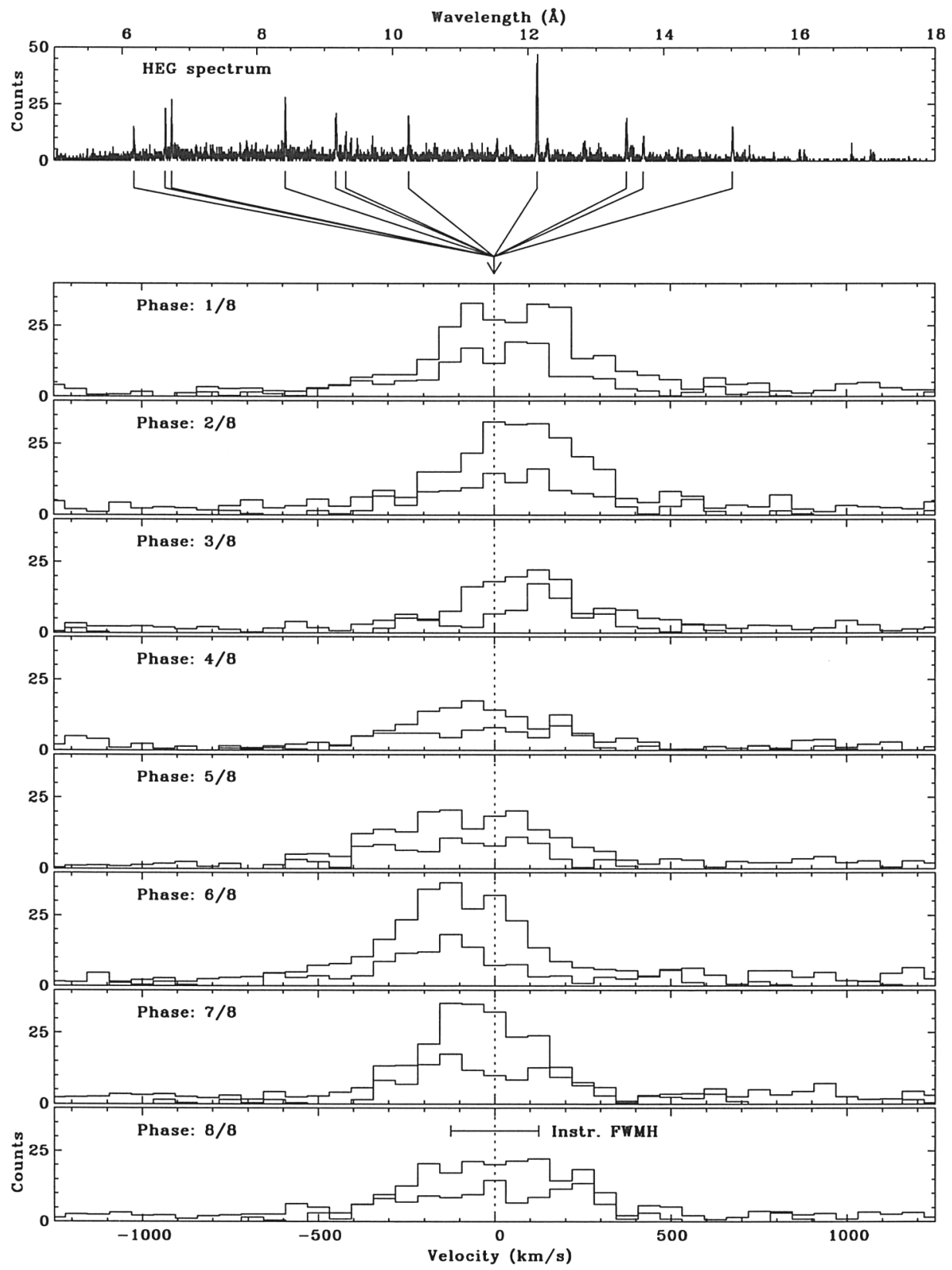

Figure 5. Line profiles for 44i Boo HETG/ACIS-S observation at different phases, showing the signal-to-noise advantage of a composite line profile (CLP) formed by summing profiles of many lines. The upper figure shows the HEG spectrum, with lines to be summed. The lower figure shows the CLPs (upper curve) in comparison with the Ne X line profile, which has been scaled by a factor of two (lower curve). Profiles have been rebinned to sum in velocity units. Courtesy of R. Hoogerwerf. 
thermal emission in the radio and not necessarily likely to produce thermal X-ray emission. Other factors which distinguish stars in binaries from single stars may be evolutionary in nature. Binary systems provide numerous possibilities for testing the size and location of the X-ray emitting region. We have discussed a number of observations from the recent literature.

The hot emission from the most active stars seems to represent a new phenomenon not easily described by solar analogies. Either X-ray emitting stellar loops are large and extended, or small and dense. In the first case they have highly expanding cross sections, unlike loops observed routinely on the Sun. In this case, it is somewhat surprising that such large loops in close binaries do not interact in some gross, observable way that would distinguish their X-ray emission properties from those of single stars with similar rotational velocities. On the other hand, if the loops are small and dense, they require large confining magnetic fields in the corona and additional physics not included in the current generation of loop models.

Of course, both scenarios are possible. While extremely extended emission is by no means ruled out observationally, there is a growing body of evidence, at least for rapidly rotating systems, for small ( $<1$ stellar radius) active regions near the poles. A predominance of small structures would also support the weak influence of binarity on coronal structure, since more compact structures are less likely to be influenced by the magnetic fields from a star's binary partner.

Acknowledgments. The author acknowledges collaborators Andrea Dupree and Ronnie Hoogerwerf, among many others. Support for this project is provided by NASA NAS8-39083 to SAO for the CXC and LTSA NAG5-3559.

\section{References}

Audard, M., Güdel, M., \& Skinner, S. L. 2003, ApJ, 589, 983

Beasley, A. J., \& Güdel, M. 2000, ApJ, 529, 967

Bingham, R., Cairns, R. A., \& Kellett, B. J. 2001, A\&A, 370, 1000

Brickhouse, N. S. 1996, in IAU Colloq. 152, Astrophysics in the Extreme Ultraviolet, ed. S. Bowyer \& R. F. Malina (Dordrecht: Kluwer), 105

Brickhouse, N. S. 2002, in ASP Conf. Ser. Vol. 277, Stellar Coronae in the Chandra and XMM-Newton Era, ed. F. Favata \& J. J. Drake, (San Francisco: ASP), 13

Brickhouse, N. S., \& Drake, J. J. 2000, in Conf. Ser. Vol. 9, Rev. Mex. de Astronomia y Astrofisica, (Mexico City), 24

Brickhouse, N. S., Dupree, A. K., \& Young, P. R. 2001, ApJ, 562, L75

Canizares, C. R. et al. 2000, ApJ, 539, L41

Choi, C. S., \& Dotani, T. 1998, ApJ, 492, 761

Drake, J. J., Brickhouse, N. S., Kashyap, V., Laming, J. M., Huenemoerder, D. P., Smith, R. K., \& Wargelin, B. J. 2001, ApJ, 548, L81

Drake, J. J., Laming, J. M., \& Widing, K. G. 1995, ApJ, 443, 393

Dupree, A. K., Brickhouse, N. S., Doschek, G. A., Green, J. C., \& Raymond, J. C. 1993, ApJ, 418, L41 
Favata, F., \& Micela, G. 2003, Space Sci.Rev., in press

Favata, F., \& Schmitt, J. H. M. M. 1999, A\&A, 350, 900

Franciosini, E., Randich, S., \& Pallavicini, R. 2003, A\&A, in press

Griffiths, N. W., \& Jordan, C. 1998, ApJ, 497, 883

Güdel, M. 1994, ApJS, 90, 743

Güdel, M., Arzner, K., Audard, M., \& Mewe, R. 2003, A\&A, 403, 155

Hoogerwerf, R., Brickhouse, N. S., \& Dupree, A. K. 2003, AAS, HEAD, 35, 12.05

Huenemoerder, D. P., Canizares, C. R., Drake, J. J., \& Sanz-Forcada, J. 2003, ApJ, submitted

Huenemoerder, D. P., Canizares, C. R., \& Schulz, N. S. 2001, ApJ, 559, 1135

Johnson, O., Drake, J. J., Kashyap, V., Brickhouse, N. S., Dupree, A. K., Freeman, P., Young, P. R., \& Kriss, G. A. 2002, ApJ, 565, L97

Lestrade, J. F., Mutel, R. L., Preston, R. A., \& Phillips, R. B. 1988, ApJ, 328, 232

Mutel, R. L., Lestrade, J. F., Preston, R. A., \& Phillips, R. B. 1985, ApJ, 289, 262

Ness, J.-U., Brickhouse, N. S., Drake, J. J., \& Huenemoerder, D. P. 2003, ApJ, accepted

Pye, J. P., Hodgkin, S. T., Stern, R. A., \& Stauffer, J. R. 1994, MNRAS, 266, 798

Raymond, J. C. \& Doyle, J. G. 1981, ApJ, 247, 3776

Reale, F., Betta, R., Peres, G., Serio, S., \& McTiernan, J. 1997, A\&A, 325, 782

Sanz-Forcada, J., Brickhouse, N. S., \& Dupree, A. K. 2003, ApJS, 145, 147

Schmitt, J. H. M. M., \& Favata, F. 1999, Nature, 401, 44

Schrijver, C. J., Lemen, J. R., \& Mewe, R. 1989, ApJ, 341, 484

Siarkowski, M., Pres, P., Drake, S. A., White, N. E., \& Singh, K. P. 1996, ApJ, 473,470

Smith, R. K., Brickhouse, N. S., Liedahl, D. A., \& Raymond, J. C. 2001, ApJ, 556, L91

Stern, R. A., Schmitt, J. H. M. M., \& Kahabka, P. T. 1995, ApJ, 448, 683

Uchida, Y., \& Sakurai, T. 1985, in Unstable Current Systems and Plasma Instabilities in Astrophysics, D. Reidel Publishing Co., Dordrecht, IAU Symp. 107,281

van den Oord, G. H. J., \& Mewe, R. 1989, A\&A, 213, 245 


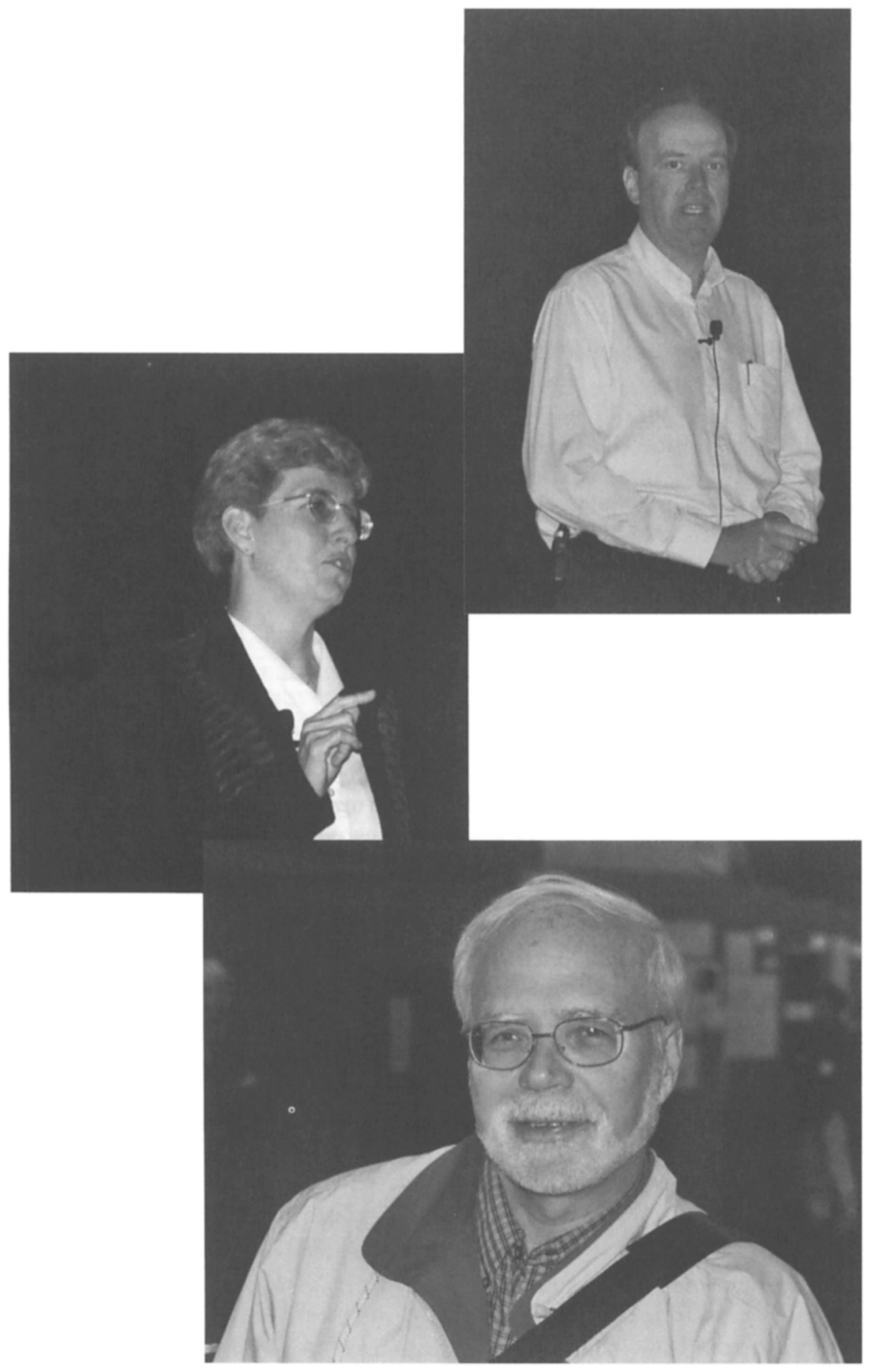

